\title{
Une nouvelle voie de transduction du FGF dans les cellules neuronales
}

Les FGF(fibroblast growth factors), identifiés grâce à leur activité mitogénique, sont aussi très étudiés pour leurs propriétés neurotrophiques. En effet, ils induisent la différenciation et la survie de certains neurones. L'unité 450 de l'Inserm s'intéresse depuis longtemps au rôle des FGF1 et FGF2 dans la rétine: (1) pendant le développement de l'œil; (2) dans la physiologie de la vision et (3) lors de maladies neurodégénératives. De très nombreuses études fondées sur des expériences in vivo et in vitro ont montré que l'addition de ces FGF prolongeait la survie des neurones de la rétine. La présence dans la rétine de FGF1 et FGF2 et de différents récepteurs de forte affinité (FGFR1 et FGFR2) et de faible affinité (HSPG) suggère l'existence probable de boucles autocrines utilisant les FGF diffusibles pour activer les récepteurs membranaires et transmettre un signal. Cependant, FGF1 et FGF2 n'ont pas de peptide signal et leur sécrétion reste encore l'objet de controverses. Or, nous avions démontré précédemment que l'expression du FGF1 dans les neurones rétiniens augmente avec la différenciation et reste élevée chez l'adulte alors qu'il ne peut avoir un rôle mitogène dans ce contexte. Cela a conduit à étudier plus précisément la transmission du signal FGF dans les cellules PC12, une lignée issue d'un phéochromocytome, utilisée classiquement comme modèle de différenciation neuronale pour étudier les mécanismes inducteurs des facteurs de croissance ou des facteurs neurotrophiques.

Sous l'effet du FGF2 ou du NGF (mais pas de l'EGF), ces cellules se différencient en étendant un réseau de neurites. Jusqu'à présent, il sem- blait établi que la différenciation des PC12 nécessitait une activation prolongée de la voie des MAP kinases ce que l'on n'obtenait qu'avec NGF ou FGF2. En fait, cette activation n'est pas suffisante pour induire la différenciation [1]. Tout d'abord, le FGF1 seul, sans héparine, et un FGF dont le site de liaison à l'héparine est muté sont capables d'activer toute la voie des MAP kinases dans des cellules PC12 sans conduire à la différenciation. A la recherche d'une autre voie, il a été démontré que seul l'ajout de FGF2 ou de FGF1 muté et d'héparine à ces cellules, induit la différenciation et une augmentation précoce de l'expression de FGF1 et FGF2.

La transfection des PC12 avec l'ADNc du FGF1, placé sous le contrôle de promoteurs constitutifs ou inductibles, a permis d'étudier la relation directe entre cette synthèse endogène des FGF1 et FGF2 et le phénotype différencié. Dans ces deux cas, on a observé une différenciation des PC12 sans signaux extérieurs. Ces expériences démontrent qu'une augmentation d'un facteur 4 ou 5 de la concentration intracellulaire de FGF1 est suffisante pour induire la différenciation sans activer apparemment une boucle autocrine. D'ailleurs, cette différenciation est obtenue en l'absence d'activation de la voie des MAP kinases. La transmission du signal neurotrophique du FGF passe donc par une nouvelle voie qui implique une néosynthèse des FGF endogènes. Cette voie est spécifique de ces facteurs puisque le NGF qui a le même effet sur ces cellules n'induit pas d'augmentation de la synthèse du FGF mais de celle du NGF. Tout se passe comme si chaque facteur neurotrophique induisait sa propre production. Il est particulièrement important de déterminer quelles sont les voies qui sont alors activées par l'augmentation endogène de ces facteurs. Dans le cadre de recherches sur l'activité mitogénique du FGF, de nombreux travaux, en particulier ceux des équipes d'Amalric et de Prats (Toulouse, France) [2], ont démontré que le FGF2, après internalisation, ou le FGF2 endogène peuvent avoir une localisation nucléaire et régler directement l'activité nucléaire. Une autre équipe (en Finlande) a également démontré que l'effet mitogénique du FGF pouvait aussi être dépendant de son internalisation [3]. Enfin, récemment, un récepteur de la famille des FGF-R à activité tyrosine kinase, mais sans signal peptide et sans domaine transmembranaire, a été découvert dans le noyau [4].

Tous ces résultats indiquent qu'il existe probablement, pour un même facteur, une dualité de fonctions: sécrété, il servirait de signal diffusible dans la communication intercellulaire; dans le noyau, il serait directement impliqué dans le contrôle du phénotype. La meilleure illustration de ce dernier point est que F. Renaud et al. (Paris, France) [1], en augmentant seulement par un facteur 4 ou 5 la concentration intracellulaire de FGF1 dans les cellules PC12 transfectées, permettent à celles-ci d'acquérir un potentiel de survie extraordinaire dans un milieu sans sérum par rapport aux PC12 d'origine. Ces résultats incitent à rechercher la voie activée directement par les FGF endogènes mais aussi à developper une stratégie moléculaire pour augmenter directement la syn- 
thèse endogène des FGF dans les neurones dans le but de prévenir les maladies dégénératives de la rétine. Y.C.

1. Renaud F, Desset S, Oliver L, Gimenez-Galego G, Van Oberghen E, Courtois Y, Laurent M. The neurotrophic activity of FGF1 depends on FGF1 expression and is independent of the MAP kinase cascade pathway. J Biol Chem 1996 ; 271 : 2801-11.

2. Amalric F, Bouche G, Bonnet H, Brethenou P

Roman AM, Truchet I, Quarto N. Fibrobast

Growth Factor 2 (FGF2) in the nucleus : translocation

process and targets. Biochem Pharmacol 1994 ;

$47: 111-5$

3. Wiedlocha A, Falnes PO, Rapak A, Munoz R,

Klingenberg O, Olsnes S. Stimulation of proliferation

of a osteosarcoma cell line by exogenous

acidic growth factor requires both activation of receptor

tyrosine kinase and growth factor internalization.

Mol Cell Biol 1996 ; 16 : 270-80.

4. Johnston Cox CH, Gomm JJ, Coombes RC. Fibroblast

Growth Factor Receptors (FGFRs) localize

in different cellular compartments. $J$ Biol Chem

1995 ; 270 : 30643-50. 